\title{
Intact Bilateral Resting-State Networks in the Absence of the Corpus Callosum
}

\author{
J. Michael Tyszka, ${ }^{1}$ Daniel P. Kennedy, ${ }^{2}$ Ralph Adolphs, ${ }^{1,2,3}$ and Lynn K. Paul ${ }^{2}$ \\ ${ }^{1}$ Division of Biology, ${ }^{2}$ Division of Humanities and Social Sciences, and ${ }^{3}$ Computation and Neural Systems Program, California Institute of Technology, \\ Pasadena, California 91125
}

Temporal correlations between different brain regions in the resting-state BOLD signal are thought to reflect intrinsic functional brain connectivity (Biswal et al., 1995; Greicius et al., 2003; Fox et al., 2007). The functional networks identified are typically bilaterally distributed across the cerebral hemispheres, show similarity to known white matter connections (Greicius et al., 2009), and are seen even in anesthetized monkeys (Vincent et al., 2007). Yet it remains unclear how they arise. Here we tested two distinct possibilities: (1) functional networks arise largely from structural connectivity constraints, and generally require direct interactions between functionally coupled regions mediated by white-matter tracts; and (2) functional networks emerge flexibly with the development of normal cognition and behavior and can be realized in multiple structural architectures. We conducted resting-state fMRI in eight adult humans with complete agenesis of the corpus callosum (AgCC) and normal intelligence, and compared their data to those from eight healthy matched controls. We performed three main analyses: anatomical region-of-interest-based correlations to test homotopic functional connectivity, independent component analysis (ICA) to reveal functional networks with a data-driven approach, and ICA-based interhemispheric correlation analysis. Both groups showed equivalently strong homotopic BOLD correlation. Surprisingly, almost all of the group-level independent components identified in controls were observed in AgCC and were predominantly bilaterally symmetric. The results argue that a normal complement of resting-state networks and intact functional coupling between the hemispheres can emerge in the absence of the corpus callosum, favoring the second over the first possibility listed above.

\section{Introduction}

How is information processed by the brain so as to generate cognition, behavior, and conscious experience? Two fundamental features of the brain's functional architecture are a rich endogenous dynamics (Raichle et al., 2001) and an organization of functional networks (Sporns et al., 2004). The first feature emphasizes the fact that, although there are covariances between brain activity and sensory input, these are generally smaller than the interregional covariances within the brain. Resting-state functional connectivity has been widely used to show that there is a set of functional networks that can be found reproducibly in healthy humans with normal cognition (Greicius et al., 2003; Damoiseaux et al., 2006; Biswal et al., 2010). Moreover, these functional networks show systematic changes during development (Supekar et al., 2009) and individual differences associated with intellect (van den Heuvel et al., 2009).

\footnotetext{
Received March 22, 2011; revised Aug. 1, 2011; accepted Aug. 18, 2011.

Author contributions: J.M.T., D.P.K., R.A., and L.K.P. designed research; J.M.T. performed research; J.M.T. analyzed data; J.M.T., D.P.K., R.A., and L.K.P. wrote the paper.

This work was supported by the Simons Foundation (SFARI-07-01 to R.A.), the National Institute of Mental Health (R01 MH080721 to R.A.), and the National Alliance for Research on Schizophrenia and Depression (2009 Young Investigator Award to L.K.P.). We thank Pratik Mukherjee, Elliott Sherr, and Leo Li for helpful discussions regarding this work. We also thank the participants for their invaluable contribution to this research.

The authors declare no competing financial interests.

Correspondence should be addressed to Dr. Michael Tyszka, 2A Broad 114-96, California Institute of Technology, 1200 E California Blvd, Pasadena, CA 91125. E-mail: jmt@caltech.edu.

DOI:10.1523/JNEUROSCI.1453-11.2011

Copyright $\odot 2011$ the authors $\quad 0270-6474 / 11 / 3115154-09 \$ 15.00 / 0$
}

The second feature raises two related questions: what are the functional networks, and how do they arise? Theoretically, one would expect the set of functional networks to correspond to the brain's repertoire of cognitive states, although this would, of course, depend on the spatiotemporal grain at which such networks can be described (He and Raichle, 2009). One might also expect that the number of functional networks exceeds that of structurally defined networks, given that brain function is dynamic and that the number of cognitive states has no obvious upper bound. These considerations raise the possibility that resting-state networks (RSNs) are supported by a variety of underlying structural bases, provided that cognition and behavior are largely normal.

We tested this latter hypothesis by imaging at rest highfunctioning adults with complete congenital absence (agenesis) of the corpus callosum (AgCC), a condition that can feature profound abnormalities in white matter connectivity despite largely normal intellect, cognition, and behavior (Paul et al., 2007). Resting-state blood oxygenation level-dependent (rsBOLD) contrast fMRI data were acquired from eight subjects with complete AgCC and a control group of eight neurologically healthy adults of comparable age, gender, and IQ. These data were analyzed using two related but complementary approaches. First, an atlasbased region-of-interest (ROI) functional connectivity analysis was performed and the regional temporal correlations compared between groups. Second, group-level independent component analyses (ICA) were performed separately for the control and AgCC subjects and the resulting spatial maps were matched and 
a



d
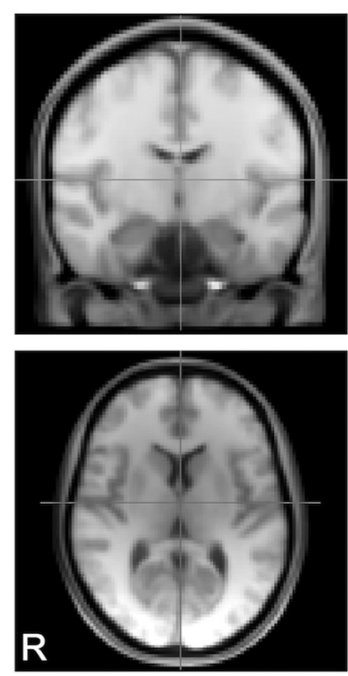

b



e
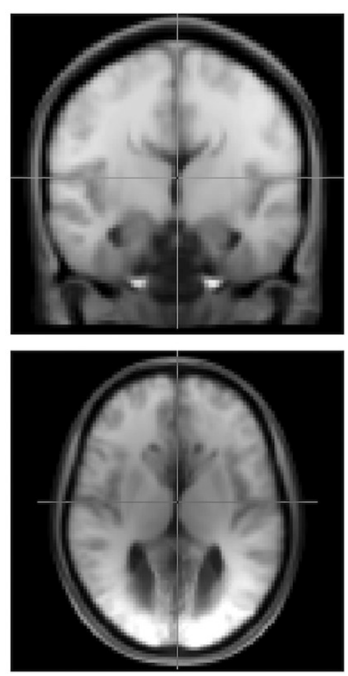

C

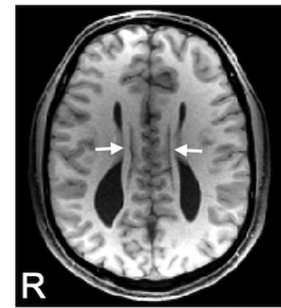

f
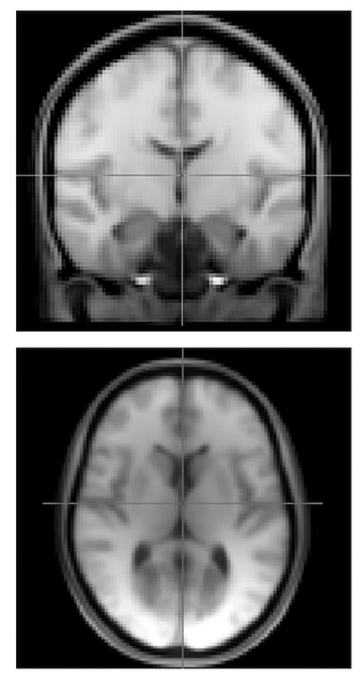

Figure 1. $\boldsymbol{a}, \boldsymbol{b}$, Representative parasagittal anatomy in control (a) and $\operatorname{AgCC}(\boldsymbol{b})$ subjects $(x=-6.0 \mathrm{~mm}) . \boldsymbol{c}, \ln \mathrm{AgCC}$, callosal fibers fail to cross midline during development, instead forming Probst bundles (white arrows, $z=+20 \mathrm{~mm}$ ). $\boldsymbol{d}-\boldsymbol{f}$, A combined midspace template was constructed by iterative nonlinear registration to an initial MNI-space target template of all T1-weighted structural images from both $\mathrm{AgCC}$ and control subjects: mean control contribution (d), mean AgCC contribution (e), and combined control and $\mathrm{AgCC}$ midspace templates $(\boldsymbol{f})$.

compared across groups. There is substantial literature support for group ICA of rsBOLD data, allowing direct comparison between our results and reproducible brain networks reported previously (Calhoun et al., 2001; Damoiseaux et al., 2006; Smith et al., 2009). Finally, we specifically queried the interhemispheric functional connectivity between ROIs defined on the basis of the ICA results with temporal correlation analysis. While related to ICA, temporal correlation analysis provides conceptually different results (Joel et al., 2011) and provided a valuable complement to data-driven ICA in specifically testing the strength of functional coupling between the two cerebral hemispheres.

\section{Materials and Methods}

Subjects. Adults with complete, isolated agenesis of the corpus callosum ( $n=8$; five males; three females; mean age, $28.1 \pm 8.0$ years) but otherwise intact anterior and posterior commissures, and matched healthy control subjects ( $n=8$; five males; three females; mean age, $27.3 \pm 8.2$ years) were recruited using a protocol approved by the Institutional Review Board of the California Institute of Technology. All subjects were fully right-handed [as measured by the short Edinburgh Handedness Questionnaire (Oldfield, 1971)] and groups did not differ in full-scale IQ $\left(t_{(14)}=1.38, p=0.83\right)$, age $\left(t_{(14)}=1.38, p=0.19\right)$, or gender ratio $\left(\chi^{2}(1)=0\right)$.

Imaging. All MRI data were acquired using a 3 tesla Magnetom Trio (Siemens Medical Solutions) with an eight-channel phased-array head receive coil and body coil transmission. Subjects were asked to lie still within the magnet with eyes closed, think of nothing specifically and to stay awake; all participants were accustomed to the scanner environment. Postscan questioning confirmed a state of relaxed wakefulness in all par- ticipants. Two T1-weighted MP-RAGE volumetric datasets were acquired as structural references $(\mathrm{TR}=1590 \mathrm{~ms}$; $\mathrm{TE}=2.7 \mathrm{~ms}$; $\mathrm{TI}=$ $800 \mathrm{~ms}$; 1 average; isotropic voxel size $=1 \mathrm{~mm}$; total imaging time $=7 \mathrm{~min}, 26 \mathrm{~s}$ ). The restingstate image acquisition consisted of $5 \mathrm{~min}$ of T2*-weighted single-shot EPI $(\mathrm{TR}=2000$; $\mathrm{TE}=30 \mathrm{~ms}$; flip angle $=75^{\circ} ; 2 \mathrm{D}$ multislice acquisition with $3.5 \mathrm{~mm}$ isotropic voxels; fat suppression; slices angled $30^{\circ}$ axial-coronal relative to the main field). Gradient echo field mapping data were acquired with identical geometry to the EPI data for EPI off-resonance distortion correction $(\mathrm{TR}=600$; $\mathrm{TE}=3.6 \mathrm{~ms}$; $6.1 \mathrm{~ms}$; flip angle $=60^{\circ}$ ).

Midspace template construction. The morphological differences between neurotypical and acallosal brains are minimal on the lateral cortical surfaces, but are pronounced around the midline and ventricles, including absence of the corpus callosum, presence of Probst bundles, mesial cortical reorganization, and enlarged posterior lateral ventricles (colpocephaly) (Fig. 1b,c). We constructed a structural midspace template by iterative nonlinear registration of all subjects' T1-weighted structural images to a reference template. In the initial pass, both control and acallosal structural data were registered using FNIRT (Andersson et al., 2008) to the MNI152 $1 \mathrm{~mm}$, whole-head, T1-weighted template. The average of all registered volumes became the template for the second pass. This process was repeated for a total of four passes, resulting in a midspace closely aligned to the MNI152 space that was used in the subsequent voxelwise ICA and anatomical ROI segmentation analyses. The local deformation required to map individual spaces onto the midspace was estimated from the determinant of the Jacobian of the warp field for each subject.

Preprocessing. Preprocessing was performed using FEAT (v5.98) and MELODIC (v3.10), within FSL (FMRIB's Software Library; www.fmrib. ox.ac.uk/fsl). Transient artifacts were removed using ArtRepair (Mazaika et al., 2005) with rigid-body motion correction, slice-timing correction, field map-based geometric distortion correction, nonbrain removal, grand-mean intensity normalization, and high-pass temporal filtering $(\sigma=50 \mathrm{~s})$. No spatial smoothing was performed at this stage. Offresonance geometric distortions in the EPI data were corrected using the PRELUDE and FUGUE toolboxes in FSL, using $B_{0}$ field maps derived from the dual-echo gradient echo dataset acquired with identical slice angle and voxel size to the EPI data. Nonbrain voxels were then masked, the data were demeaned, and variance normalized on a voxelwise basis. BOLD EPI data were registered to the midspace template in two steps: an initial affine registration to the T1-weighted structural image, using the magnitude data from the field map as a proxy for the EPI space; and a nonlinear registration of the T1-weighted structural image to the midspace template. EPI data were resampled in the midspace at an isotropic spatial resolution of $4 \mathrm{~mm}$. The same preprocessed data were used for both group-level ICA and region-based correlation analysis.

Atlas-based interregional correlation analysis. Functional connectivity reflected by temporal BOLD signal correlation was analyzed on signal time-series extracted from anatomically defined regions, together covering the entire cortex in each subject. Gray matter, white matter, and CSF partial volume estimates (PVEs) were generated from each subject's T1weighted structural data using the FSL FAST tool (Zhang et al., 2001) and transformed to the midspace. For each subject, nuisance signals derived from white matter and CSF time courses were removed from the preprocessed BOLD time series using a general linear model (GLM) approach, as described previously (Fox et al., 2009; Anderson et al., 2011). The 
white matter regressor was highly correlated with whole-brain signal $\left(R^{2}\right.$ typically $>0.95$, $p<0.0001)$ and is functionally equivalent to global signal regression. The GLM was constructed with regressors corresponding to mean signal within a deep white matter ROI, a ventricular CSF ROI, and the six rigid body motion correction parameter time courses. The residual of this model, subsequently referred to as the corrected BOLD signal, was then used for interregional correlation analysis. Cortical regions were defined probabilistically using the Harvard-Oxford Atlas, colocalized to the anatomical midspace used in this study. A joint probabilistic mask was formed from the product of the individual gray matter PVE and atlas cortical region and used to weight the calculation of the first temporal eigenvariate from each subject's preprocessed BOLD time course data. The use of the first eigenvariate instead of the weighted spatial mean to represent a region's BOLD time course has been previously justified on the basis of reduced sensitivity to signal heterogeneity within the region (Friston et al., 2006).

Pearson's product-moment correlation coefficients were calculated for all pairs of atlas ROI time courses, resulting in an upper triangle symmetric interregional correlation matrix for each subject. All correlation coefficients within the matrix were Fisher $z$-transformed before subsequent statistical analysis. Group differences between the Fisher $z$ matrices in their entirety were assessed by $t$ tests of each matrix element using false discovery rate (FDR) correction $(q=0.05)$. In addition, the 48 homotopic elements were extracted from the correlation matrix and assessed for group differences with a two-way ANOVA and post hoc $t$ tests with FDR correction. Effect sizes are reported as Cohen's $d$ for unpaired $t$ tests and partial $\eta^{2}\left(\eta_{p}^{2}\right)$ for ANOVA.

Independent component analysis. Independent spatiotemporal components of the BOLD signal were isolated for the AgCC and control groups separately using temporally concatenated probabilistic independent component (IC) analysis (Calhoun et al., 2001). A preliminary single-subject ICA-based analysis was performed to identify artifactual cardiac and respiratory components. For each subject's dataset, ICs with $>50 \%$ of the estimated spectral power in frequencies $>0.1 \mathrm{~Hz}$ or with $>25 \%$ of signal in white matter or CSF were removed from the time series, resulting in a cleaned, prefiltered dataset. Probabilistic principal component analysis (PCA) was used to estimate the number of ICs to decompose (Beckmann and Smith, 2004). The whitened observations were decomposed into sets of vectors that describe signal variation across the concatenated subject-time domain, and across the spatial domain by optimizing for non-Gaussian spatial source distributions. Estimated IC spatial maps were normalized to the standard deviation of the residual noise and thresholded at a false-discovery rejection rate of 95\% for visualization purposes by fitting a mixture model to the histogram of intensity values (Beckmann and Smith, 2004). ICs associated with motion-by-magnetic field interactions at tissue-bone or tissue-air interfaces, or which were localized primarily in the white matter or CSF spaces, were classified as artifactual and excluded from further analysis.

The remaining nonartifactual ICs in the AgCC group were then matched to the IC with the highest spatial correlation coefficient in the deformation in $\mathrm{AgCC}$ (Fig. 6). b

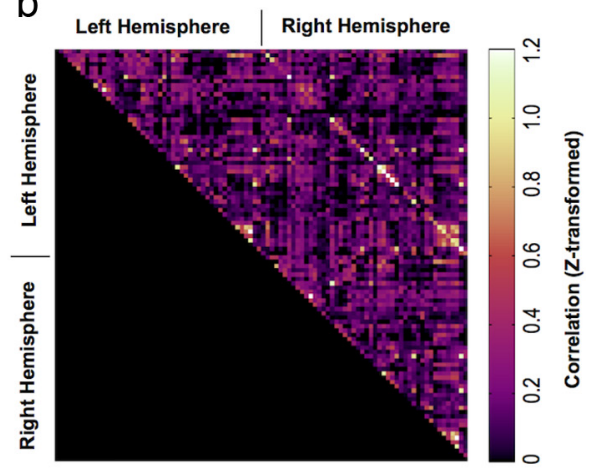

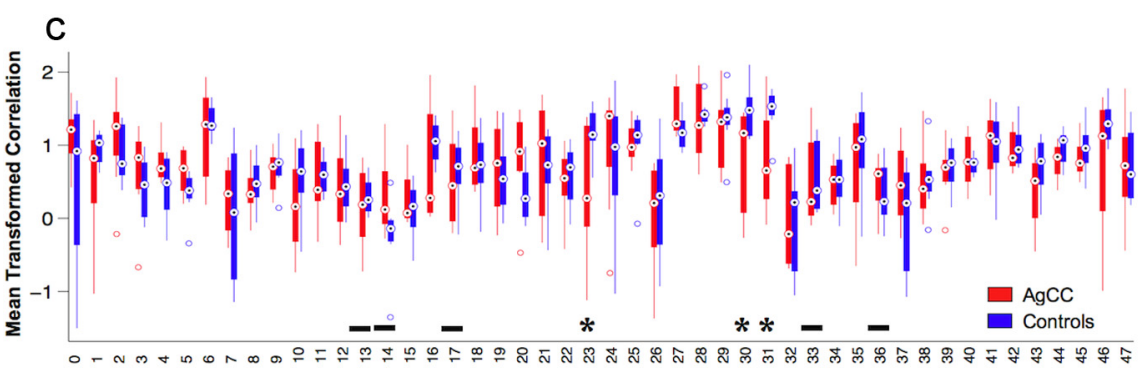

Harvard-Oxford Atlas Cortical Region
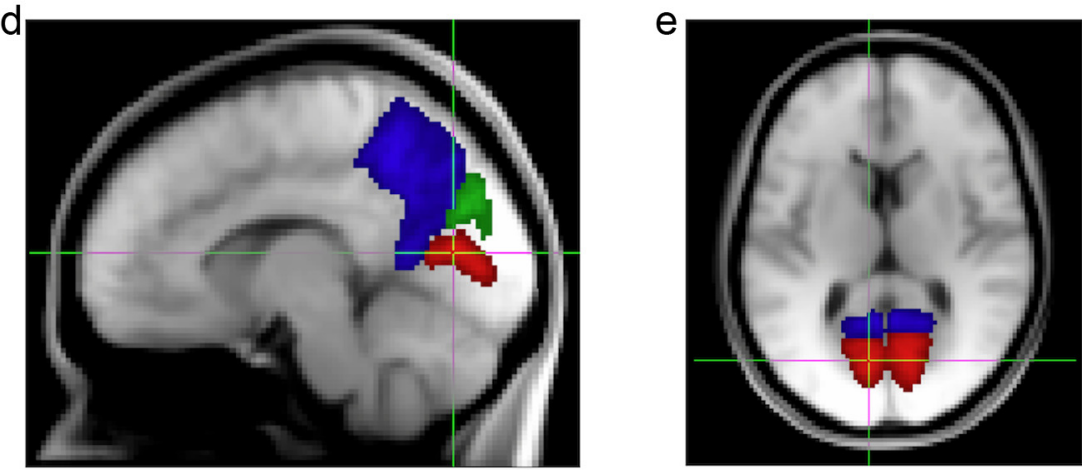

Figure 2. $\quad \boldsymbol{a}, \boldsymbol{b}$, Mean interregional correlations in BOLD signal from 48 Harvard-0xford atlas labels in both hemispheres for regions with reduced functional correlation in $\mathrm{AgCC}$ identified in c. These regions correspond to areas of significantly increased local

control group, constrained to within-brain voxels by the group-level brain mask. IC spatial maps were not thresholded for spatial correlation calculations. The group-level mask excluded a small portion of the most dorsal parietal cortex that was not sampled in all AgCC subjects due to the larger average brain volume in this group.

Two measures of similarity between groups of spatial independent components have been described in the context of BOLD fMRI data (Varoquaux et al., 2010), specifically a measure of the overall stability of the decomposition between two groups, $e$, and the degree of one-to-one reproducibility in paired ICs, $t$. Two groups of spatial ICs, each with $m$ voxels and $n_{1}$ and $n_{2}$ components, are represented respectively as $m \times n_{1}$ and $m \times n_{2}$ matrices, $\mathbf{A}_{1}$ and $\mathbf{A}_{2}$ with unit-normed columns. Defining the intergroup correlation matrix as $\mathbf{C}=\mathbf{A}_{1}{ }^{\mathrm{T}} \mathbf{A}_{2}$, the intergroup stability can then be defined as the normalized energy of the cross-correlation matrix

$$
e=\frac{1}{d} \operatorname{tr}\left(\mathbf{C}^{T} \mathbf{C}\right)
$$

where $\operatorname{tr}(\cdot)$ is the trace of the matrix and $d=\min \left(\operatorname{rank} \mathbf{A}_{1}, \operatorname{rank} \mathbf{A}_{2}\right)$. 
The one-to-one reproducibility of matched pairs of spatial ICs, $t$, is estimated from the reduced cross-correlation matrix, $\tilde{\mathbf{C}}$, composed only of the coefficients of the invertible IC pairings, as proposed by Varoquaux et al. (2010).

$$
t=\frac{1}{d} \operatorname{tr}(\tilde{\mathbf{C}})
$$

Pairings were considered invertible if and only if they met the following criteria: for two IC spatial maps, A1 and A2, from groups 1 and 2, respectively, A2 must have the highest spatial correlation of all group 2 ICs with A1 and A1 must have the highest spatial correlation of all group 1 ICs with A2. Invertible pairings can be identified from the correlation matrix $\mathrm{C}$ as elements that have the highest correlation coefficient in both a row and a column.

Interhemispheric temporal correlation of group independent components. The temporal correlation of the corrected BOLD signal (defined previously for atlas-based analysis) was calculated between left and right hemisphere ROIs defined by the suprathreshold voxel masks of each group-level IC. Each group was analyzed separately. The left and right hemisphere principal eigenvariate signals for each IC ROI were extracted as before and the Fisher $z$-transformed correlation coefficient calculated between left and right hemisphere eigenvariates. Between-group comparisons were performed for interhemispheric Fisher $z$ values using masks from all neuronal ICs (unpaired $t$ test, two-tailed, unequal variance) and from each invertible IC pairing (paired $t$ test, two-tailed, unequal variance, FDR corrected, $q=0.05$ ). The symmetry of each group IC mask was defined as the percentage overlap of the reflection through midline of the left hemisphere mask and the right hemisphere mask. IC symmetry was compared between groups for all neuronal ICs (unpaired $t$ test) and between invertible IC matches (paired $t$ test). Effect sizes are reported as Cohen's $d$ for unpaired $t$ tests, Pearson's $r$ for paired $t$ tests, and $\eta_{p}^{2}$ for ANOVA.

\section{Results}

Atlas-based BOLD correlation

Interregional correlation of the principal temporal eigenvariates from the 48 gray-matter weighted atlas regions revealed strong homotopic correlations in both control and AgCC groups (Fig. $2 a, b)$. No significant differences were observed between any element of the Fisher $z$-transformed correlation matrix of the AgCC and control groups following FDR correction $(q=0.05)$. Differences in the $z$-transformed correlation assessed by a two-way ANOVA were highly significant between regions $\left(F_{(47,672)}=6.53\right.$, $\left.p<0.00001, \eta_{p}^{2}=0.314\right)$ and nonsignificant between groups $\left(F_{(1,672)}=0.76, p=0.385, \eta_{p}^{2}=0.001\right)$, with a nonsignificant region-by-group interaction $\left(F_{(47,672)}=1.33, p=0.074, \eta_{p}^{2}=\right.$ $0.085)$. Only three homotopic pairings - the intracalcarine, precuneus, and cuneal cortices (Harvard-Oxford atlas labels 23, 30, and 31) - showed significant uncorrected differences $\left(t_{(14)}=\right.$ $2.38,2.27,2.54 ; p=0.032,0.040,0.023 ; d=1.27,1.21,1.36$, respectively) in correlation between AgCC and controls (Fig. $2 c-$ e). In each case, the interregional correlation was lower in AgCC than in controls, though none of the differences survived false discovery rate correction $(q=0.05)$. Some atlas regions were partially clipped by the group-level calculation mask, which excluded regions of very low signal or incomplete coverage. Homotopic pairs with $>50 \%$ clipping in one or both regions are indicated with a solid black bar in Figure $2 c$. All but one of these regions lay in the inferior temporal cortex. The correlation comparisons for these clipped regions should be considered as preliminary.

\section{Independent component analysis}

For the first-level single-subject ICA, the average dimensionality (actual number of independent components) was estimated by

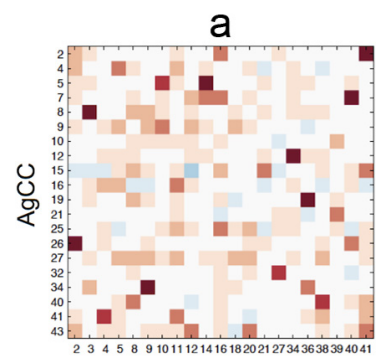

Controls

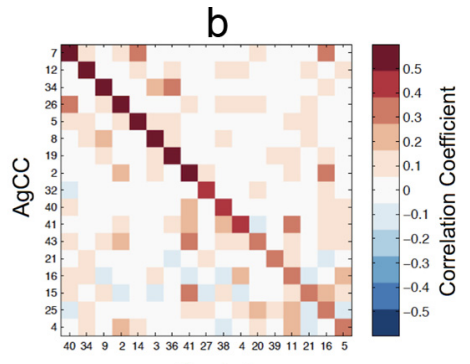

Controls
Figure 3. Spatial correlation matching of group-level ICs. $\boldsymbol{a}$, Full correlation matrix, C, for all neuronal ICs identified in the ICA of control and $\mathrm{AgCC}$ groups. Searching rows and columns for the maximum correlation coefficient identifies candidate pairings of ICs from each group. A pairing is defined as invertible if the corresponding matrix element contains the maximum correlation in both row and column. $\boldsymbol{b}$, Reduced correlation matrix, $\tilde{\mathbf{C}}$, composed of the reordered invertible IC pairings.

PCA to be $52.8 \pm 2.8$ (mean \pm SEM) for AgCC and $44.3 \pm 9.6$ for controls. On average, $21.4 \pm 3.7$ of AgCC ICs and $16.5 \pm 3.7$ of control ICs were classified automatically, based on power spectrum and tissue compartment, as artifactual and removed at this level. No significant differences were observed between the AgCC and control groups on total IC count $\left(t_{(14)}=1.931, p=0.074\right.$, $d=1.03)$ and artifactual IC count $\left(t_{(14)}=0.939, p=0.364, d=\right.$ 0.50). For the second-level group ICA, 20 of 44 AgCC ICs and 21 of 41 control ICs were classified as neuronal, accounting for $53.8 \%$ and $62.7 \%$ of the total variance, respectively. Thus, overall, the numbers of total, artifactual, and neural ICs, as well as the proportion of variance in BOLD signal finally accounted for, were very comparable between AgCC and control datasets.

We paired neuronal ICs between the two groups using correlation analysis of the spatial IC modes (without thresholding) and identified 17 invertible matches (and two noninvertible but likely matches) between AgCC and control ICs from a total of 20 and 21 neuronal ICs in the respective groups (Figs. 3, 4). The correlation coefficients between the spatial modes in each invertible pairing ranged from 0.328 to 0.695 ( $n=109,350$ voxels total, $p \ll 0.0001$ in all cases). We further characterized the correspondence between ICs in the AgCC and control groups and found high measures of intergroup stability $(e=0.40)$ and one-to-one paired reproducibility $(t=0.41)$. These values are lower than those observed for intersession comparisons in resting state $(e=0.58$ and $t=0.51)$ but comparable to localizer tasks $(e=0.43$ and $\mathrm{t}=$ 0.35 ) reported previously in healthy subjects (Varoquaux et al., 2010). The variance explained by each of the neuronal ICs in Figure 4, $a$ and $b$, is summarized in Table 1 .

Consistent RSNs have been identified by BOLD fMRI across healthy adult subjects in several independent studies (Greicius et al., 2003; Damoiseaux et al., 2006; Fox et al., 2007; Smith et al., 2009; Biswal et al., 2010; Varoquaux et al., 2010) and many of these ICs are reproduced in both the control and AgCC groups here. For example, Smith et al. (2009) identifies 10 networks that are reproducibly isolated in both a resting-state study $(n=26)$ and a task-based BOLD fMRI meta-analysis $(n=29,671)$ using a 20-component ICA. All 10 of these networks are reproduced in both the control and AgCC groups of this study, either as single components or decomposed into subnetworks (Fig. 4, Table 1). Two potential pairs of components, namely the bilateral motor network (A9 and C10) and a bilateral parietal network (A27 and C18), were not immediately identified using our invertibility criteria but could be matched by inspection (Fig. 4b). Two control (C8 and C12) and one $\mathrm{AgCC}$ (A10) component had no corresponding match in the 
a

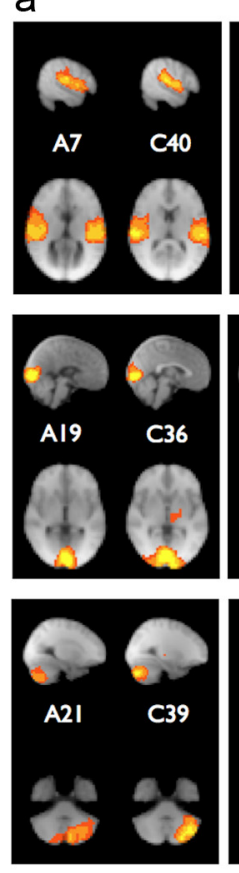

b

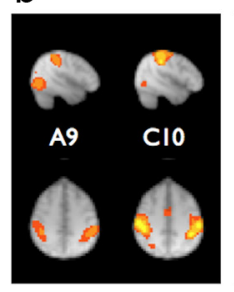

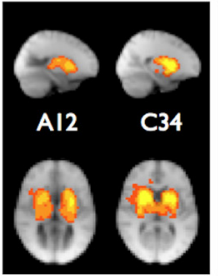
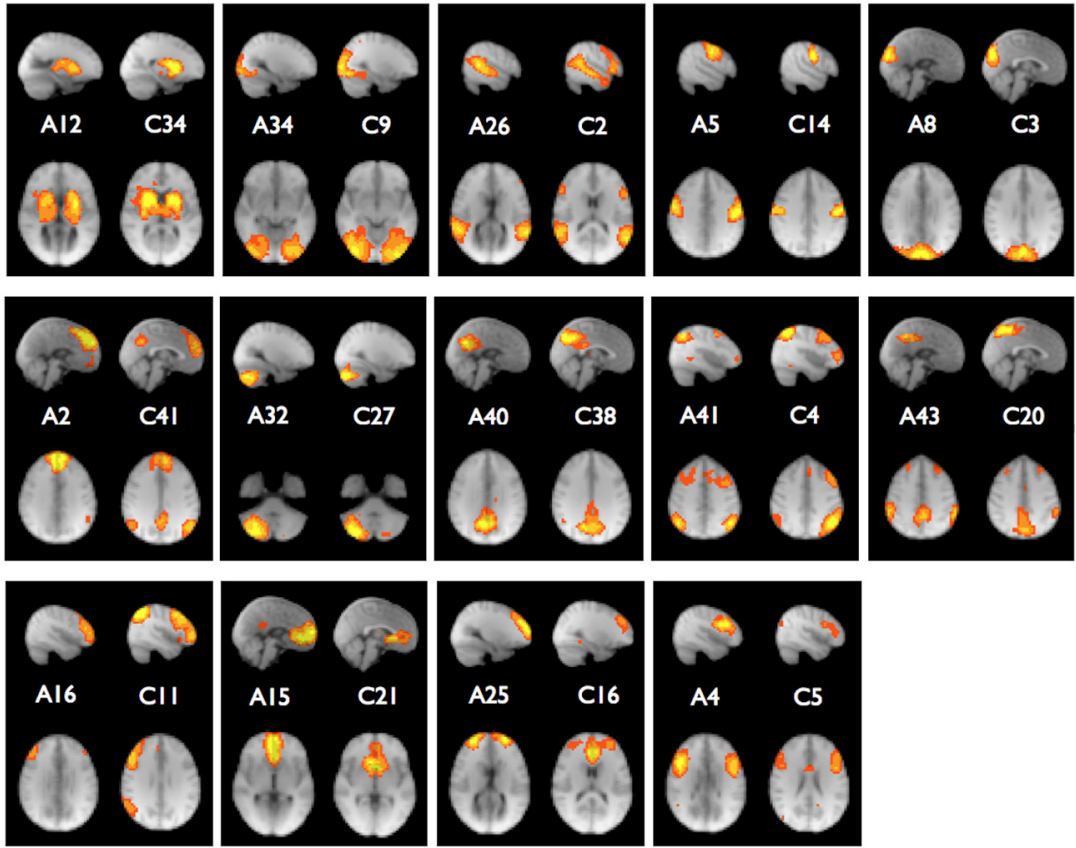

C

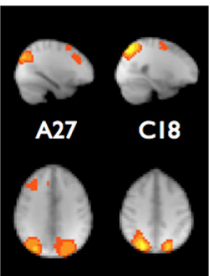

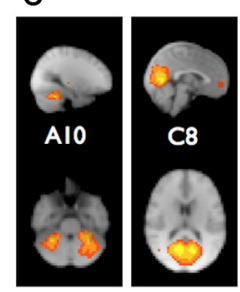

d

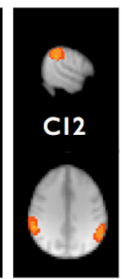

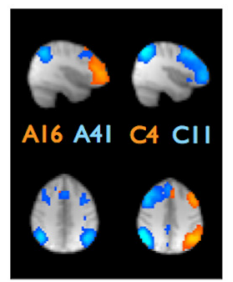

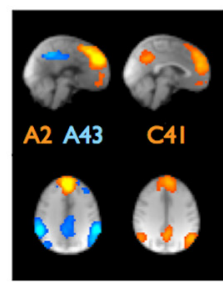

Figure 4. $\boldsymbol{a}$, Invertible matches between AgCC and control group independent component spatial maps, ordered by decreasing spatial correlation coefficient from highest (A7-C40, $r=0.695)$ to lowest (A4-C5, $r=0.328)$. $\boldsymbol{b}$, Additional pairings (by inspection) from the remaining ICs without invertible matches. $\boldsymbol{c}$, Unmatched ICs: a bilateral cerebellar component in AgCC (A10) and a medial occipital (C8) and lateral parietal (C12) component in controls. $d$, Suboptimal invertible matches occur when networks in either group are split into subcomponents by the ICA. For example, the dorsal parietal-frontal networks are lateralized in control subjects (C4 and C11) but split anterior-posterior in AgCC (A16 and A41). Similarly, the default mode network is a single component in the control group (C41), but split into frontal and parietal components in $\mathrm{AgCC}$ (A2 and A43).

other group (Fig. 4c). The dorsal parietal-frontal network, observed as left- and right-lateralized components in controls $(\mathrm{C} 4, \mathrm{C} 11)$, is split anterior-posterior in AgCC (A16, A41) (Fig. 4d). Similarly, the default mode network appears as two components in AgCC (A2, A43), but one in controls (C41). The tentative functional assignment, variance explained, spatial correlations, and correspondence to literature networks are summarized in Table 1.

\section{Interhemispheric temporal correlation for group-level} independent components

No overall difference was seen in the interhemispheric correlations within group IC mask ROIs between the AgCC and control groups (unpaired $t$ test, $p=0.778$ ) (Fig. 5a). Differences in the $z$-transformed interhemispheric temporal correlation of the invertible group IC pairs assessed by two-way ANOVA were highly significant between regions $\left(F_{(16,238)}=3.28, p=0.00001, \eta_{p}^{2}=\right.$ $0.180)$ and nonsignificant between groups $\left(F_{(1,238)}=0.41, p=\right.$ $\left.0.523, \eta_{p}^{2}=0.002\right)$, with a significant region-by-group interaction $\left(F_{(16,238)}=2.09, p=0.01, \eta_{p}^{2}=0.123\right)$. Only one invertible pair (A21 and C39, left cerebellum) showed a significant difference in interhemispheric temporal correlation $\left(t_{(14)}=5.828, p=\right.$ $0.003, d=3.12$ ) (Fig. $5 b$ ). It should be noted that only one suprathreshold voxel was present in the right hemisphere mask for C39 and is the most likely explanation for the low interhemi- spheric correlation for this IC and the significant difference with the A21 correlation score. Symmetry scores were relatively high, with a median of $\sim 60 \%$ in each group (Fig. $5 c$ ). No significant between-group difference was seen in the symmetry of neuronal group ICs $\left(t_{(39)}=0.739, p=0.464, d=0.237\right)$ or in the symmetry of invertible group IC pairs $\left(t_{(16)}=1.943, p=0.070, r=0.437\right)$. However, several group ICs have symmetry scores $<20 \%$ (Fig. $5 d$ ), including the left and right cerebellar ICs seen in both groups, and left- and right-lateralized components of the dorsal parietal-frontal network seen in control subjects.

Morphological variability and independent component differences The morphological differences between AgCC and control brains raise the concern that differences in local deformation during spatial registration could bias functional analyses. We constructed a metric of absolute change in volume from the local Jacobian, $J$, of the warp transformation required to map an individual brain onto the midspace template: $\delta=|\log [\operatorname{det}(J)]|$. The $\log$ of the determinant (det) of $J$ is zero when there is no local volume change and taking the absolute value prevents cancellation during integration of positive and negative volume changes within a region of interest. The mean value of $\delta$ was calculated over all subjects within each group-level IC spatial mask for the control and AgCC groups; results are summarized in Figure 6. 
Table 1. Correspondences of group-level AgCC neuronal independent components to control group and literature components

\begin{tabular}{|c|c|c|c|c|c|}
\hline IC number & Best control IC match & Correlation coefficient & Explained variance & Assignment & Corresponding IC in Smith et al. (2009) \\
\hline \multicolumn{6}{|l|}{ Invertible } \\
\hline A7 & $\mathrm{C} 40$ & 0.695 & $4.28 \%$ & Auditory (frontal) & $7_{20}$ \\
\hline $\mathrm{A} 12$ & C34 & 0.689 & $3.48 \%$ & Caudate-putamen & - \\
\hline A34 & C9 & 0.654 & $0.82 \%$ & Ventral visual & $3_{20}$ \\
\hline A26 & $\mathrm{C} 2$ & 0.595 & $1.47 \%$ & Auditory (temporal) & $7_{20}$ \\
\hline A5 & $\mathrm{C} 14$ & 0.586 & $4.70 \%$ & Premotor & - \\
\hline A8 & $\mathrm{C} 3$ & 0.548 & $4.10 \%$ & Visual & $1_{20}$ (part) \\
\hline A19 & $\mathrm{C} 36$ & 0.527 & $2.73 \%$ & Visual & 220 \\
\hline $\mathrm{A} 2$ & C41 & 0.497 & $5.61 \%$ & DMN (with A43) & $4_{20}$ \\
\hline A32 & $\mathrm{C} 27$ & 0.476 & $0.88 \%$ & Cerebellum (right) & $5_{20}$ (part) \\
\hline A40 & $\mathrm{C} 38$ & 0.424 & $0.04 \%$ & Posterior cingulate & $4_{20}$ (part) \\
\hline A41 & C4 & 0.407 & $0.08 \%$ & Parietal-frontal (with A16) & $9_{20}, 10_{20}$ \\
\hline A43 & $\mathrm{C} 20$ & 0.382 & $0.67 \%$ & DMN (with A19) & $4_{20}$ \\
\hline A21 & $\mathrm{C} 39$ & 0.348 & $2.49 \%$ & Cerebellum (left) & $5_{20}$ (part) \\
\hline A16 & $\mathrm{C} 11$ & 0.346 & $3.09 \%$ & Parietal-frontal (with A41) & $9_{20}, 10_{20}$ \\
\hline A15 & $\mathrm{C} 21$ & 0.339 & $3.20 \%$ & vmPFC & $8_{20}$ (part) \\
\hline A25 & $\mathrm{C} 16$ & 0.332 & $1.88 \%$ & Frontal pole & $8_{20}$ (part) \\
\hline A4 & $C 5$ & 0.328 & $4.79 \%$ & dIPFC & - \\
\hline \multicolumn{6}{|c|}{ Noninvertible } \\
\hline A9 & $\mathrm{C} 10$ & & $4.09 \%$ & Somatomotor & $6_{20}$ \\
\hline A10 & - & & $3.96 \%$ & Cerebellum & $5_{20}$ (part) \\
\hline A27 & $\mathrm{C} 18$ & & $1.43 \%$ & $\mathrm{~d} I O C$ & - \\
\hline Total & & & $53.79 \%$ & & \\
\hline
\end{tabular}

Seventeen of the 20 components were matched invertibly to control ICs and two noninvertible components were matched by inspection (A9 and A27). DMN, Default mode network; vmPFC, ventromedial prefrontal cortex; dIPFC, dorsolateral prefrontal cortex; dIOC, dorsolateral occipital cortex.

For this purpose, ICs A16 + A41, A2 + A43, and C4 + C11 were treated as single ICs (Fig. $4 d$ ). Two-way ANOVA showed highly significant main effect of group $\left(F_{(1,266)}=77.69, p<0.0001\right.$, $\left.\eta_{p}^{2}=0.226\right)$, IC pair $\left(F_{(18,266)}=11.93, p<0.0001, \eta_{p}^{2}=0.447\right)$, and group-by-IC pair interaction $\left(F_{(18,266)}=6.39, p<0.0001\right.$, $\left.\eta_{p}^{2}=0.302\right)$ attributable to three pairs: A8/C3, A19/C36, and A40/C38 $\left(t_{(14)}=4.68,6.11,10.17 ; p<0.001\right.$ in each case, $d=$ 1.67, 2.40, 2.00; respectively; post hoc Bonferroni-corrected $t$ test).

\section{Discussion}

Our study produced two broad sets of findings. First, anatomical ROI-based analyses of BOLD correlations showed strong functional connectivity between homotopic cortical brain regions in the AgCC group, and this did not differ significantly in any region from the correlations found in the control group. The temporal BOLD correlation between regions defined by the left and right spatial modes of the group independent components was also essentially unaffected by the absence of the corpus callosum. Second, resting-state networks identified in the AgCC group were very similar to those identified in the control group and correspond to networks consistently identified in the resting-state literature in healthy adult subjects (Table 1). Together, these findings provide substantial support for the hypothesis that identical resting-state functional networks can be realized on different structural architectures, at least in a developmental context that permits substantial reorganization.

Strikingly, a high level of bilateral symmetry was observed in the spatial ICs of the AgCC group, equivalent to that found in controls. Previous rsBOLD fMRI studies in healthy adults have shown a high degree of interhemispheric correlation that was significantly greater between homotopic regions than between heterotopic regions (Stark et al., 2008; Toro et al., 2008), a pattern reproduced in our results. The level of bilateral IC symmetry in the AgCC group together with the entirely intact functional cor- relation between homotopic cortical regions demonstrates unambiguously that interhemispheric functional integration can occur in the absence of the corpus callosum.

Interhemispheric functional synchronization in the absence of direct callosal connectivity has been reported in other species that lack a corpus callosum, such as song birds (Schmidt, 2003). Schmidt (2003) proposed that intrinsic motor networks in the song bird were likely synchronized by inputs from the thalamus or midbrain, a mechanism that is also plausible for the network symmetry observed here in human AgCC subjects. In a recent study in monkeys (Matsui et al., 2011), intrinsic functional connectivity (inFC) in rsBOLD data were compared directly to effective functional connectivity inferred from evoked BOLD responses to electrical microstimulation (emFC). While intrahemispheric inFC and emFC were strongly correlated, interhemispheric emFC correlation was substantially lower than inFC, suggesting that inFC may reflect a network-level synchronization not captured by emFC. The surprisingly normal interhemispheric functional connectivity we report here in AgCC may thus arise from one or both of these factors: common subcortical drivers or complex network-level synchronization, neither of which requires direct structural connectivity between cortical components.

The decomposition of the ventral frontal-parietal network into left- and right-lateralized components has been reported previously in healthy subjects, for example as patterns $C$ and $D$ in Damoiseaux et al. (2006) and components $9_{20}$ and $10_{20}$ in Smith et al. (2009). The appearance of these two networks as a single, bilateral component in AgCC may result from an artifactual integration by the ICA. The decomposition of the default mode network into frontal and parietal components in the AgCC group may be similarly artifactual in a cohort of this size. The reliability of these two findings would need to be tested in a larger sample allowing more fine-grained, higher dimensionality ICA to be performed with confidence. At this stage, we do not draw any conclusions regarding differences in the ICAs observed between the 
two groups and restrict ourselves to a focus on their much more striking commonalities.

Our results initially appear to conflict with three sets of findings in the literature. First, it has been noted that functional and structural connectivity are in fact correlated in healthy brains (Greicius et al., 2009). However, this does not necessarily contradict our results, since those correlations were modest and indirect structural and functional connectivity were also correlated $(r=0.29)$ (Honey et al., 2009). Thus, structural connectivity only partially shapes functional organization, consistent with the expected greater number and flexibility of functional networks compared with structural networks that we noted earlier. Our findings suggest that normal resting-state functional networks and largely normal cognition can emerge even under rare circumstances where the structural base is disrupted.

Second, a pediatric case study reported a dramatic reduction in functional connectivity between homotopic cortical regions following callosotomy to treat severe epilepsy (Johnston et al., 2008). However, callosal disconnection is congenital in our subjects and the transection was acute in the case study (Johnston et al., 2008). While this provides an important contrast to our results, two other case reports demonstrate relatively preserved resting interhemispheric correlation in split-brain patients (Corsi-Cabrera et al., 1995; Uddin et al., 2008), suggesting possible compensatory mechanisms.

Third, a case study of three patients with AgCC and a single neurotypical control (Quigley et al., 2003) concluded that resting-state functional connectivity in the auditory and motor cortices was significantly diminished in AgCC. Although this case study is a valuable contribution to the literature on functional connectivity in callosal agenesis, the present study uses more participants and therefore may give a more generalizable account of functional connectivity in AgCC.

We note that differences between AgCC and control groups in both homotopic correlations (Fig. 2) and regional deformation (Fig. 6) are significant in posterior midline regions. It may well be that abnormal anatomy in posterior midline cortices in AgCC results in abnormal processing within these structures. It is intriguing to note that some of the putative roles of posterior cingulate and retrosplenial cortices correspond to functions known to be impaired in AgCC, namely self-relevant cognition and social functions (Paul et al., 2007). The posterior midline in AgCC remains a target for further study using more detailed cortical segmentation approaches (Fischl et al., 1999; Destrieux et al., 2010).

RSNs are known to be present in infancy (Fransson et al., 2011) and even at birth (Doria et al., 2010) when the corpus callosum has its adult complement of axons but is not yet fully myelinated (Luders et al., 2010), and show progressive changes a

b

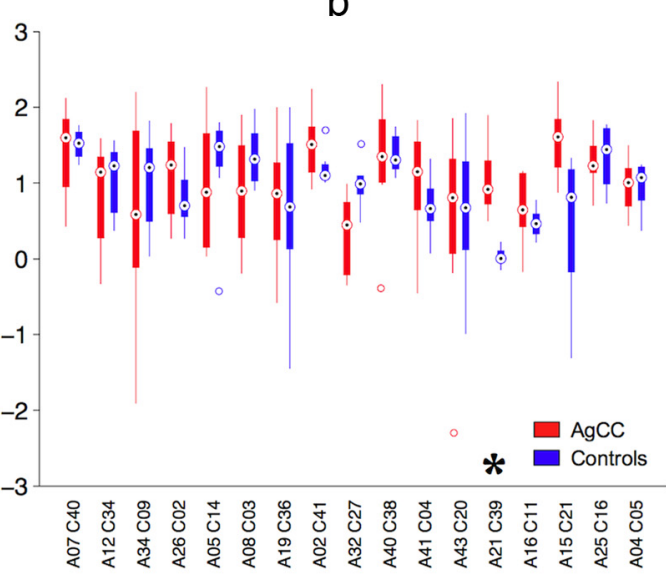

C



Figure 5. Interhemispheric temporal BOLD correlations for each of the group-level ICS. Masks were defined within the left and right hemispheres for suprathreshold voxels for each group-level IC. Extracted BOLD time series (corrected for white matter and CSF (a) were correlated and mean Fisher z-transformed correlation calculated for all subjects. $\boldsymbol{a}$, No significant difference was be groups in an unpaired comparison of all neuronal ICs $(p=0.778) . \boldsymbol{b}$, Interhemispheric correlations for the ble IC pairs from each group. Only one IC pair showed a significant difference in interhemispheric correlation ( ${ }^{*} \mathrm{~A} 21-\mathrm{C} 39$, left . 0.026 FR corrected). c, Left-right percentage symmetry of the group IC masks used for calculating the BOLD temporal correlations in $\boldsymbol{a}$ and $\boldsymbol{b}$ did not differ between groups (for symmetry definition, see Materials and Methods). $\boldsymbol{d}$, Left-right percentage symmetry for the ICs that comprise the 17 invertible IC pairings.

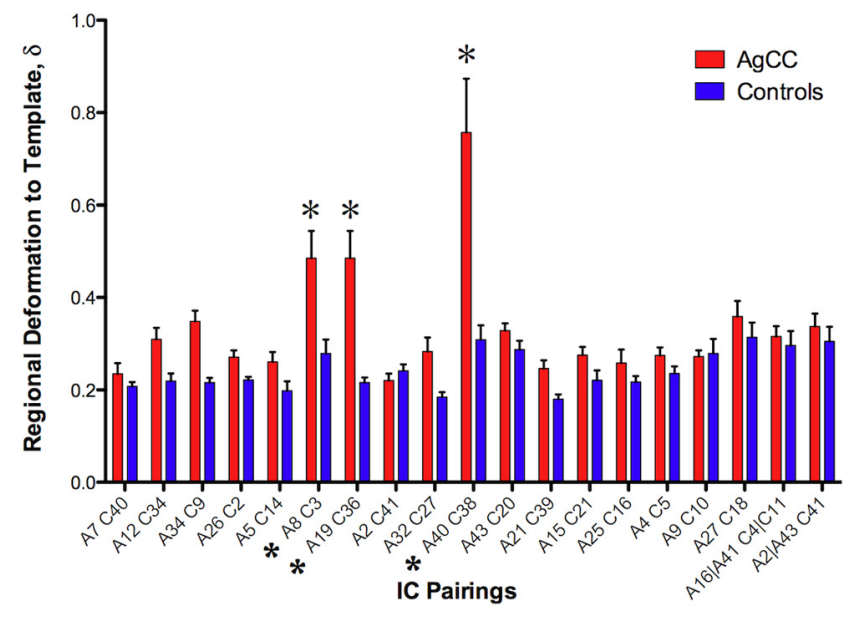

Figure 6. Mean absolute deformation within suprathreshold regions for each of the $19 \mathrm{IC}$ pairs illustrated in Figure 4. The combination of A16 and A41 has been paired with C 4 and C11, as has $\mathrm{A} 2$ and $\mathrm{A} 43$ with C41, as described in the Results, Morphological variability and independent component differences. IC pairs with significantly higher regional deformation in AgCC compared with controls $\left(^{*}\right)$ all correspond to posterior midline locations, which also show significantly reduced homotopic temporal correlation (Fig. 2c). 
throughout childhood (Supekar et al., 2009), concomitant with changes in structural connectivity (Hagmann et al., 2010) and cognitive development (Fair et al., 2009). It is unknown whether typical RSNs are present in AgCC infants. Functional brain networks derived from rsBOLD data are known to bear important relationships to cognition: they are disrupted in neurodegenerative diseases and dementia (Stam et al., 2007) and they correlate with IQ in healthy adults (van den Heuvel et al., 2009). All of these findings suggest that, just as structural connectivity forms the normal basis for functional networks, the latter form the basis for emergence of cognition. Direct structural connectivity does not appear to be a strict determinant of the development of functional connectivity in healthy brains, as indicated by the present study.

The full characterization of the RSNs we describe in patients with AgCC remains an important question. Our study shows that there are surprisingly normal networks in AgCC in terms of their bilateral symmetry, number, neuroanatomical distribution, and total explained variance in BOLD signal. However, it will be necessary to quantify the relative strength of functional connectivity between network components, their spectral power distribution, and eventually their relationship to networks that subserve specific aspects of cognitive functioning. The latter issue will be of particular importance, since it is known that otherwise highfunctioning acallosal subjects (as studied here) do exhibit subtle impairments in social and executive functions, though the overt impairments classically seen in surgical commissurotomy patients are absent (Paul et al., 2007). Since RSNs comprise the set of all those networks also activated during specific tasks (Smith et al., 2009), as well as the default-mode network, the detailed pattern of ICs could provide important insights into the profile of cognitive abilities of people with AgCC.

It will be important to compare the present results with other developmental disorders known to have abnormal cognition. Notably, people with autism have reduced corpus callosum cross-sectional area (Frazier and Hardan, 2009) and fractional anisotropy (Kumar et al., 2010), show changes in the defaultmode component of RSNs (Kennedy and Courchesne, 2008), and show reduced homotopic functional connectivity consistent with reduced direct callosal coupling (Anderson et al., 2011). Strikingly, there is very recent evidence that $\sim 20-30 \%$ of people with AgCC meet criteria for a diagnosis of an autism spectrum disorder (Booth et al., 2011; Paul et al., 2011). The nature and developmental arc of resting-state functional connectivity in AgCC subjects with autism remains an open question.

The present findings argue that largely normal functional networks can emerge in brains with dramatically altered structural connectivity. This may be limited to cases where (1) the structural connectivity abnormality is developmental, permitting substantial functional reorganization early in life; and (2) where cognition is largely normal. Perhaps the most profound aspect of the present findings is the suggestion that the functional organization of the brain subserving cognition can be driven by factors other than direct structural connectivity. Determining these factors is an important topic for future investigations and may help us understand not only how the healthy human mind develops, but also suggesting strategies for intervention in developmental disorders.

\section{References}

Anderson JS, Druzgal TJ, Froehlich A, DuBray MB, Lange N, Alexander AL, Abildskov T, Nielsen JA, Cariello AN, Cooperrider JR, Bigler ED, Lainhart JE (2011) Decreased interhemispheric functional connectivity in autism. Cereb Cortex 21:1134-1146.
Andersson JL, Smith S, Jenkinson M (2008) FNIRT: FMRIB non-linear image registration tool. Paper presented at Meeting for the Organization for Human Brain Mapping, Melbourne, Australia, June.

Beckmann CF, Smith SM (2004) Probabilistic independent component analysis for functional magnetic resonance imaging. IEEE Trans Med Imaging 23:137-152.

Biswal B, Yetkin FZ, Haughton VM, Hyde JS (1995) Functional connectivity in the motor cortex of resting human brain using echo-planar MRI. Magn Reson Med 34:537-541.

Biswal BB, Mennes M, Zuo XN, Gohel S, Kelly C, Smith SM, Beckmann CF, Adelstein JS, Buckner RL, Colcombe S, Dogonowski AM, Ernst M, Fair D, Hampson M, Hoptman MJ, Hyde JS, Kiviniemi VJ, Kötter R, Li SJ, Lin CP, et al (2010) Toward discovery science of human brain function. Proc Natl Acad Sci U S A 107:4734-4739.

Booth R, Wallace GL, Happé F (2011) Connectivity and the corpus callosum in autism spectrum conditions Insights from comparison of autism and callosal agenesis. Prog Brain Res 189:303-317.

Calhoun VD, Adali T, Pearlson GD, Pekar JJ (2001) A method for making group inferences from functional MRI data using independent component analysis. Hum Brain Mapp 14:140-151.

Corsi-Cabrera M, Trías G, Guevara MA, Haro R, Hernández A (1995) EEG interhemispheric correlation after callosotomy: one case study. Percept Mot Skills 80:504-506.

Damoiseaux JS, Rombouts SA, Barkhof F, Scheltens P, Stam CJ, Smith SM, Beckmann CF (2006) Consistent resting-state networks across healthy subjects. Proc Natl Acad Sci U S A 103:13848-13853.

Destrieux C, Fischl B, Dale A, Halgren E (2010) Automatic parcellation of human cortical gyri and sulci using standard anatomical nomenclature. Neuroimage 53:1-15.

Doria V, Beckmann CF, Arichi T, Merchant N, Groppo M, Turkheimer FE, Counsell SJ, Murgasova M, Aljabar P, Nunes RG, Larkman DJ, Rees G, Edwards AD (2010) Emergence of resting state networks in the preterm human brain. Proc Natl Acad Sci U S A 107:20015-20020.

Fair DA, Cohen AL, Power JD, Dosenbach NU, Church JA, Miezin FM, Schlaggar BL, Petersen SE (2009) Functional brain networks develop from a "local to distributed" organization. PLoS Comput Biol 5:e1000381.

Fischl B, Sereno MI, Dale AM (1999) Cortical surface-based analysis. II. Inflation, flattening, and a surface-based coordinate system. Neuroimage 9:195-207.

Fox MD, Snyder AZ, Vincent JL, Raichle ME (2007) Intrinsic fluctuations within cortical systems account for intertrial variability in human behavior. Neuron 56:171-184.

Fox MD, Zhang D, Snyder AZ, Raichle ME (2009) The global signal and observed anticorrelated resting state brain networks. J Neurophysiol 101:3270-3283

Fransson P, Aden U, Blennow M, Lagercrantz H (2011) The functional architecture of the infant brain as revealed by resting-state FMRI. Cereb Cortex 21:145-154.

Frazier TW, Hardan AY (2009) A meta-analysis of the corpus callosum in autism. Biol Psychiatry 66:935-941.

Friston KJ, Rotshtein P, Geng JJ, Sterzer P, Henson RN (2006) A critique of functional localisers. Neuroimage 30:1077-1087.

Greicius MD, Krasnow B, Reiss AL, Menon V (2003) Functional connectivity in the resting brain: a network analysis of the default mode hypothesis. Proc Natl Acad Sci U S A 100:253-258.

Greicius MD, Supekar K, Menon V, Dougherty RF (2009) Resting-state functional connectivity reflects structural connectivity in the default mode network. Cereb Cortex 19:72-78.

Hagmann P, Sporns O, Madan N, Cammoun L, Pienaar R, Wedeen VJ, Meuli R, Thiran JP, Grant PE (2010) White matter maturation reshapes structural connectivity in the late developing human brain. Proc Natl Acad Sci U S A 107:19067-19072.

He BJ, Raichle ME (2009) The fMRI signal, slow cortical potential and consciousness. Trends Cogn Sci 13:302-309.

Honey CJ, Sporns O, Cammoun L, Gigandet X, Thiran JP, Meuli R, Hagmann P (2009) Predicting human resting-state functional connectivity from structural connectivity. Proc Natl Acad Sci U S A 106:2035-2040.

Joel SE, Caffo BS, van Zijl PC, Pekar JJ (2011) On the relationship between seed-based and ICA-based measures of functional connectivity. Magn Reson Med 66:644-657.

Johnston JM, Vaishnavi SN, Smyth MD, Zhang D, He BJ, Zempel JM, Shi- 
mony JS, Snyder AZ, Raichle ME (2008) Loss of resting interhemispheric functional connectivity after complete section of the corpus callosum. J Neurosci 28:6453-6458.

Kennedy DP, Courchesne E (2008) The intrinsic functional organization of the brain is altered in autism. Neuroimage 39:1877-1885.

Kumar A, Sundaram SK, Sivaswamy L, Behen ME, Makki MI, Ager J, Janisse J, Chugani HT, Chugani DC (2010) Alterations in frontal lobe tracts and corpus callosum in young children with autism spectrum disorder. Cereb Cortex 20:2103-2113.

Luders E, Thompson PM, Toga AW (2010) The development of the corpus callosum in the healthy human brain. J Neurosci 30:10985-10990.

Matsui T, Tamura K, Koyano KW, Takeuchi D, Adachi Y, Osada T, Miyashita Y (2011) Direct comparison of spontaneous functional connectivity and effective connectivity measured by intracortical microstimulation: an fMRI study in macaque monkeys. Cereb Cortex 10:2348-2356.

Mazaika PK, Whitfield S, Cooper JC (2005) Detection and Repair of Transient Artifacts in fMRI Data. Paper presented at Meeting of the Society for Human Brain Mapping, Toronto, Ontario, Canada, June.

Oldfield RC (1971) The assessment and analysis of handedness: the Edinburgh inventory. Neuropsychologia 9:97-113.

Paul LK, Brown WS, Adolphs R, Tyszka JM, Richards LJ, Mukherjee P, Sherr EH (2007) Agenesis of the corpus callosum: genetic, developmental and functional aspects of connectivity. Nat Rev Neurosci 8:287-299.

Paul LK, Corsello C, Kennedy DP, Childress D, Cheng BC, Adolphs R (2011) Autism symptomatology in primary agenesis of the corpus callosum. Paper presented at International Meeting for Autism Research, San Diego, CA, May.

Quigley M, Cordes D, Turski P, Moritz C, Haughton V, Seth R, Meyerand ME (2003) Role of the corpus callosum in functional connectivity. AJNR Am J Neuroradiol 24:208-212.

Raichle ME, MacLeod AM, Snyder AZ, Powers WJ, Gusnard DA, Shulman GL (2001) A default mode of brain function. Proc Natl Acad Sci U S A 98:676-682.

Schmidt MF (2003) Pattern of interhemispheric synchronization in HVc during singing correlates with key transitions in the song pattern. J Neurophysiol 90:3931-3949.

Smith SM, Fox PT, Miller KL, Glahn DC, Fox PM, Mackay CE, Filippini N, Watkins KE, Toro R, Laird AR, Beckmann CF (2009) Correspondence of the brain's functional architecture during activation and rest. Proc Natl Acad Sci U S A 106:13040-13045.

Sporns O, Chialvo DR, Kaiser M, Hilgetag CC (2004) Organization, development and function of complex brain networks. Trends Cogn Sci 8:418-425.

Stam CJ, Jones BF, Nolte G, Breakspear M, Scheltens P (2007) Small-world networks and functional connectivity in Alzheimer's disease. Cereb Cortex 17:92-99.

Stark DE, Margulies DS, Shehzad ZE, Reiss P, Kelly AM, Uddin LQ, Gee DG, Roy AK, Banich MT, Castellanos FX, Milham MP (2008) Regional variation in interhemispheric coordination of intrinsic hemodynamic fluctuations. J Neurosci 28:13754-13764.

Supekar K, Musen M, Menon V (2009) Development of large-scale functional brain networks in children. PLoS Biol 7:e1000157.

Toro R, Fox PT, Paus T (2008) Functional coactivation map of the human brain. Cereb Cortex 18:2553-2559.

Uddin LQ, Mooshagian E, Zaidel E, Scheres A, Margulies DS, Kelly AM, Shehzad Z, Adelstein JS, Castellanos FX, Biswal BB, Milham MP (2008) Residual functional connectivity in the split-brain revealed with restingstate functional MRI. Neuroreport 19:703-709.

van den Heuvel MP, Stam CJ, Kahn RS, Hulshoff Pol HE (2009) Efficiency of functional brain networks and intellectual performance. J Neurosci 29:7619-7624.

Varoquaux G, Sadaghiani S, Pinel P, Kleinschmidt A, Poline JB, Thirion B (2010) A group model for stable multi-subject ICA on fMRI datasets. Neuroimage 51:288-299.

Vincent JL, Patel GH, Fox MD, Snyder AZ, Baker JT, Van Essen DC, Zempel JM, Snyder LH, Corbetta M, Raichle ME (2007) Intrinsic functional architecture in the anaesthetized monkey brain. Nature 447:83-86.

Zhang Y, Brady M, Smith S (2001) Segmentation of brain MR images through a hidden Markov random field model and the expectationmaximization algorithm. IEEE Trans Med Imaging 20:45-57. 